\title{
Maternidade na adolescência: indicadores emocionais negativos e fatores associados em mães de 14 a 16 anos em Porto Alegre, RS, Brasil
}

\author{
Maternity during adolescence: negative emotional indicators \\ and associated factors in 14 to 16-year-old mothers \\ from Porto Alegre in the State of Rio Grande do Sul, Brazil
}

Micheli Scolari Rossetto ${ }^{1}$ Lígia Braun Schermann ${ }^{1}$ Jorge Umberto Béria ${ }^{1}$
${ }^{1}$ Programa de PósGraduação em Saúde Coletiva, Universidade Luterana do Brasil. Av. Farroupilha 8001/Prédio 14/ sala 228, São Luis. 92.425900 Canoas RS Brasil. michelirossetto@

hotmail.com

\begin{abstract}
The prevalence of negative emotional indicators and associated factors in 430 adolescent mothers between 14 to 16 years of age in Porto Alegre in the State of Rio Grande do Sul was detected. Socio-demographic variables, social and family relationships, reproductive aspects, abuse and violence were studied. The prevalence rate $(P R)$ was obtained by Poisson regression using hierarchical analyses. The prevalence of intense psychological distress was $32.6 \%$, and it was associated with low social class, grade repetition, bad relationship with the mother, lack of acceptance of the pregnancy by the father, and lack of family support during pregnancy. The prevalence of negative self-esteem was $15.4 \%$, and it was associated with the bad relationship with the mother and not having a person to confide in. Little or no expectation for the future was found in $7.5 \%$ of the adolescents and was associated with grade repetition, nurturing by the biological mother, early sexual initiation, and the occurrence of physical abuse. The high prevalence of psychological distress found in the adolescent mothers of this study deserves special attention from the public policies in health in order to include qualified professionals to manage the emotional aspects of early motherhood.
\end{abstract}

Key words Pregnancy in adolescence, Adolescence, Maternity, Emotional indicators, Associated factors
Resumo Verificou-se a prevalência de indicadores emocionais negativos e fatores associados em 430 mães adolescentes de 14 a 16 anos de Porto Alegre, $R S$. Foram estudadas variáveis sociodemográficas, relações sociais e familiares, aspectos reprodutivos, abuso e violência. As razões de prevalência (RP) foram obtidas por regressão de Poisson mediante análise hierarquizada. A prevalência de sofrimento psíquico intenso foi $32,6 \%$, estando associado à baixa classe social, à não repetência escolar, ao relacionamento ruim com a mãe, à não aceitação da gestação pelo parceiro e à falta de apoio da família frente à gestação. A prevalência de autovalorização negativa foi $15,4 \%$, permanecendo associada ao relacionamento ruim com a mãe e a não possuir uma pessoa confidente. Pouca ou nenhuma expectativa em relação ao futuro foi encontrada em 7,5\% das adolescentes, estando associada à repetência escolar, à criação pela mãe biológica, à idade precoce da primeira relação sexual e à ocorrência de abuso físico. A alta prevalência de sofrimento psíquico, encontrada nas mães adolescentes, merece atenção especial das políticas públicas de saúde para a inclusão de profissionais habilitados ao manejo dos aspectos emocionais no atendimento da maternidade precoce.

Palavras-chave Gravidez na adolescência, Adolescência, Maternidade, Indicadores emocionais, Fatores associados 


\section{Introdução}

Mundialmente, 16 milhões de mulheres de 15 a 19 anos engravidam a cada ano e aproximadamente $11 \%$ de todos os nascimentos concentram-se em países de baixa e média renda ${ }^{1}$. Dados do Ministério da Saúde brasileiro apontam uma diminuição significativa no número de gravidez na adolescência nas últimas duas décadas, sendo que, nos anos de 2000 a 2005, registrou-se uma diminuição de 15,6\%, e de 2005 a 2009, de $22 \%$. No entanto, apesar desta redução geral nos índices, os números ainda constituem motivo de preocupação $0^{2}$. Em estudo realizado em três capitais brasileiras com 4.634 adolescentes em idade inferior a 15 anos, as prevalências de gravidez foram 2,2\% em Porto Alegre, $1,2 \%$ no Rio de Janeiro e 3,5\% em Salvador ${ }^{3}$.

A adolescência é uma fase que se caracteriza pela transição da infância para a idade adulta, período marcado por grandes transformações físicas, cognitivas e sociais ${ }^{4}$. Uma gravidez neste período representa um fator adicional com o qual a adolescente precisa lidar. Pesquisadores destacam que as vivências emocionais da adolescente em relação à sua gravidez e maternidade têm sido apresentadas de forma heterogênea, evidenciando diferentes padrões de percepção: ora positivos com manifestações de satisfação, ganhos emocionais e afirmação da autoestima; ora negativos, com tendências à depressão e à percepção da maternidade como uma experiência difícil e solitária ${ }^{5,6}$.

As variações destas repercussões parecem estar diretamente relacionadas às condições socioeconômicas e do contexto sociocultural da mãe adolescente ${ }^{7}$. Para Duarte et al. ${ }^{8}$, em seu estudo com 1.314 adolescentes de Santo André (SP), os resultados indicaram que a maior taxa de fecundidade (35,7 em 1.000 adolescentes) esteve associada às piores condições socioeconômicas, enquanto que a menor taxa $(12,1 \mathrm{em} \mathrm{1.000)}$ foi observada em famílias com melhores condições. Outro estudo mostra que as adolescentes, que estão fora da escola quando engravidam, apresentam uma pior autovalorização e menores expectativas em relação ao futuro?.

Em relação ao contexto familiar, a literatura aponta a necessidade de a adolescente sentir-se apoiada por seus familiares durante o período gestacional ${ }^{10}$. Piccinini et al. ${ }^{11}$ investigaram o apoio social de mães adolescentes e mães adultas, da gestação ao terceiro mês de vida do bebê, mostrando que as adolescentes solicitavam mais a ajuda da família e demonstravam uma necessidade maior de receber apoio, principalmente de suas próprias mães do que as mães adultas. Outros estudos igualmente identificam as próprias mães e os parceiros como a principal fonte de apoio afetivo e material para a mãe adolescente $e^{10,12}$.

Quando há reação negativa dos familiares diante da gravidez, as adolescentes sentem-se menos valorizadas, com poucas expectativas em relação ao futuro e manifestam intenso sofrimento psíquico9. Estas vivências negativas podem afetar não só o desempenho da adolescente em sua função materna, mas a qualidade do vínculo mãe-bebê, o que representa um importante fator para o desenvolvimento infantil saudável ${ }^{13,14}$.

O presente estudo tem como objetivo verificar a prevalência de indicadores emocionais negativos (sofrimento psíquico intenso, pouca ou nenhuma expectativa em relação ao futuro e autovalorização negativa) e fatores associados (sociodemográficos, relações sociais e familiares, aspectos reprodutivos, abuso e violência) em mães adolescentes de 14 a 16 anos, que tiveram filhos no ano de 2009 em Porto Alegre, RS. Este estudo faz parte de uma pesquisa mais ampla intitulada: "Fatores associados à gestação na adolescência: Um estudo de casos e controles com jovens de 14 a 16 anos em Porto Alegre, RS" desenvolvida pelos professores do Programa de Pós-Graduação em Saúde Coletiva da Ulbra (PPGSC) e financiada pelo Conselho Nacional de Pesquisa (CNPq).

\section{Método}

A população em estudo é formada por mães adolescentes entre 14 e 16 anos, que tiveram filhos em Porto Alegre no ano de 2009. Para composição da amostra, tomaram-se como base os dados do Sistema Nacional de Nascidos Vivos (SINASC) da cidade de Porto Alegre, referente ao número de bebês nascidos vivos no ano de 2008, de mães adolescentes de 14 a 16 anos (850), estimando-se esse mesmo número de partos entre adolescentes com essas idades para o ano de 2009. Embora tenha sido planejado escolher aleatoriamente $50 \%$ das adolescentes que tiveram filho em 2009, a estratégia foi impossível porque muitos endereços não foram encontrados. Todas as mães que se enquadraram em um critério de elegibilidade para composição da amostra foram procuradas até três vezes. Quando não encontradas, foram substituídas por outra adolescente do SINASC Porto Alegre até que a amostra proposta para o estudo estivesse completa.

Os dados foram coletados mediante aplicação de questionário estruturado, com entrevis- 
tas nas residências das adolescentes, bem como informações contidas na Declaração de Nascidos Vivos (DN). A coleta aconteceu entre os meses de julho/2009 a maio/2010, por duplas de estudantes mulheres da área da saúde da Universidade Luterana do Brasil (Ulbra-Canoas) previamente treinadas e sob supervisão. Foi realizado um estudo piloto com onze mães adolescentes, que não estavam entre as incluídas na amostra, para adequação do questionário e da logística.

O Termo de Consentimento Livre e Esclarecido (TCLE) era assinado pelo(a) responsável e, em caso de emancipação da adolescente, esta assinava o TCLE. O controle de qualidade das entrevistas deu-se através de contatos telefônicos aleatórios em que algumas questões do questionário eram perguntadas novamente e checada a veracidade das respostas.

A mensuração dos fatores emocionais foi baseada em um instrumento utilizado por Sabroza et al. ${ }^{9}$, dividido em três fatores: sofrimento psíquico (12 itens), autovalorização (seis itens) e expectativa em relação ao futuro (cinco itens). Os itens são pontuados conforme uma escala Likert. O ponto de corte para autovalorização negativa foi 1,64 e pouca ou nenhuma expectativa em relação ao futuro 1,60, estipulados a partir do quartil inferior da distribuição das médias. Já o ponto de corte para sofrimento psíquico intenso foi 0,92 , equivalente ao quartil superior da distribuição das médias.

Foram estudadas variáveis sociodemográficas, referentes a relações sociais e familiares, a aspectos reprodutivos, abuso físico e abuso sexual como possíveis determinantes dos desfechos autovalorização negativa, pouca ou nenhuma expectativa em relação ao futuro e sofrimento psíquico intenso. Nas questões referentes ao abuso físico e sexual, era lida uma breve definição dos termos antes $\mathrm{da}$ adolescente responder às perguntas. Abuso físico foi considerado quando alguém usa a força física de forma intencional com possibilidade de causar ou efetivamente causando dano físico em outra pessoa e abuso sexual, quando alguém tocar as partes sexuais de uma pessoa contra sua vontade ou através de sedução ou obrigar alguém a ter relação sexual ou sexo oral através do uso da força, ameaça ou sedução ou quando alguém obriga outro a tocar nos seus genitais para satisfação própria ou quando há exploração sexual de crianças e adolescentes através de prostituição, exposição sexual ou pornografia.

O banco de dados foi construído através do programa Teleform, com escaneamento dos questionários e posterior migração dos dados para o pacote estatístico SPSS 18.0 for Windows para fins de análise. A análise estatística constou de análise univariada; análise bivariada com cruzamento das variáveis de exposição e dos indicadores emocionais mediante tabelas de contingência (teste qui-quadrado) e teste para tendência linear, e, por último, análise multivariada, realizada através de regressão de Poisson com variância robusta para a investigação do efeito conjunto das variáveis de exposição sobre os indicadores emocionais. Este teste foi escolhido por se tratar de estudo transversal com desfecho não raro $^{15}$.

A análise multivariada foi realizada conforme modelo teórico hierarquizado, expresso na Figura 1, que permite verificar se a associação entre os indicadores emocionais e o fator em estudo é direta ou mediada pelo efeito das outras variáveis. Os efeitos das variáveis que se encontram em um mesmo nível hierárquico funcionam como fatores de confusão para as demais do mesmo nível e para as de níveis inferiores. Por sua vez, estas últimas podem exercer um efeito mediador das influências das variáveis de níveis superiores.

Permaneceram no modelo as variáveis cujo valor de $p$ foi igual ou menor que 0,20 na análise bivariada. Quando eram incluídas as variáveis de blocos mais proximais ao desfecho, estas eram ajustadas para as variáveis que haviam permanecido nas análises dos blocos anteriores. Consideraram-se significativos os resultados com valores de $p<0,05$ (Figura 1).

Essa pesquisa foi elaborada de acordo com a Resolução 196/96 ${ }^{16}$ do Conselho Nacional de Saúde/Ministério da Saúde e foi aprovada pelo Comitê de Ética da Ulbra e pela Secretaria Municipal de Saúde de Porto Alegre.

\section{Resultados}

Foram entrevistadas 431 mães adolescentes. Excluiu-se uma por falecimento do bebê, restando 430 mães para análise. Considerando que a literatura apresenta prevalência de $26,8 \%$ de sofrimento psíquico intenso em mães adolescentes ${ }^{9}$, para que fosse possível a estimativa de repercussões emocionais negativas no presente trabalho com margem de erro de 5 pontos percentuais e nível de confiança de 95\% seriam necessárias 219 entrevistadas. Além disso, o poder do estudo seria de $80 \%$ para estimar razões de prevalência de 1,5 para os fatores de risco estudados, com nível de confiança de 0,05.

Os desfechos avaliados foram autovalorização $(\mathrm{n}=429)$, expectativa em relação ao futuro 


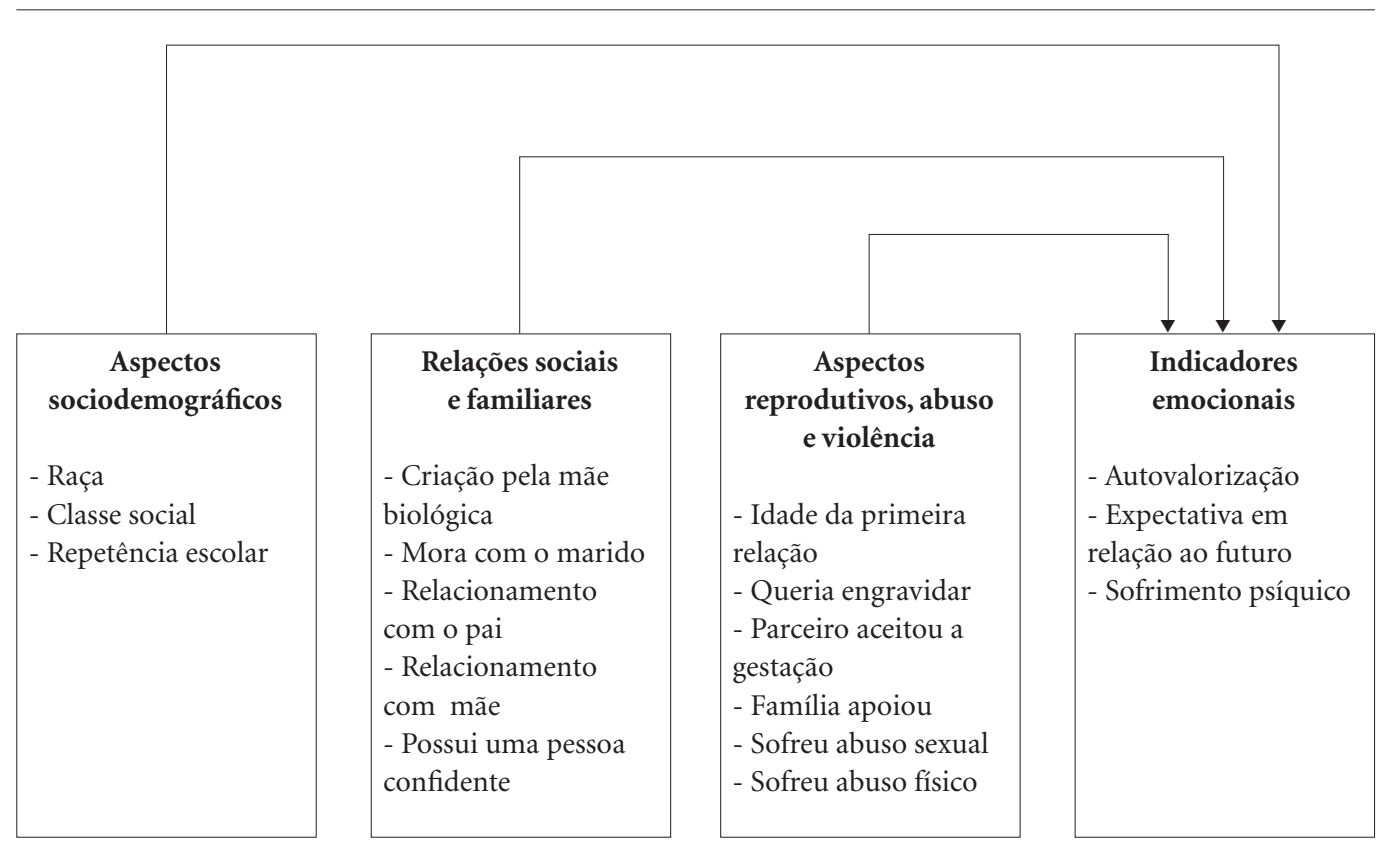

Figura 1. Modelo de análise hierarquizado de determinação da autovalorização, expectativa em relação ao futuro e sofrimento psíquico em mães adolescentes de 14 a 16 anos.

$(\mathrm{n}=428)$ e sofrimento psíquico $(\mathrm{n}=426)$. A prevalência de autovalorização negativa foi $15,4 \%$ (n =66), de pouca ou nenhuma expectativa em relação ao futuro foi $7,5 \%(n=32)$ e de sofrimento psíquico intenso foi $32,6 \%(n=139)$.

Quanto às características sociodemográficas das entrevistadas, 51\% declararam-se não brancas, 57,0\% encontravam-se na classe C (classificação socioeconômica da Abipeme) e a maioria (78\%) repetiu o ano escolar mais de uma vez. As relações sociais e familiares caracterizaram-se por $89 \%$ das adolescentes terem sido criadas pela mãe biológica, 59\% morarem com o marido, $61 \%$ terem bom relacionamento com o pai, $80 \%$ terem bom relacionamento com a mãe e $82 \%$ possuírem pelo menos uma pessoa confidente.

Em relação aos aspectos reprodutivos, a maioria (56\%) teve a primeira relação entre 13 e 14 anos, $83 \%$ não queriam engravidar, 78\% dos parceiros aceitaram a gravidez, $90 \%$ das famílias apoiaram a gestação. A grande maioria (94\%) das adolescentes nunca sofreu abuso sexual e $86 \%$ nunca sofreram abuso físico.

A Tabela 1 apresenta os resultados da associação das variáveis estudadas com sofrimento psíquico intenso. $\mathrm{Na}$ análise bivariada, as variáveis raça, classe social, repetência escolar, relacionamento com a mãe, possuir uma pessoa confiden- te, idade da primeira relação sexual, aceitação do parceiro na gestação, apoio da família na gestação e sofreu abuso físico alcançaram nível de significância observada menor a 20\%. Destas, permaneceram no modelo final da análise multivariada com significância menor que 5\%: classe social (D+E com RP = 2,29; IC95\%: 1,13-4,65), relacionamento ruim com a mãe ( $\mathrm{RP}=2,02$; IC95\%: 1,56-2,62), parceiro não aceitou a gestação (RP = 1,48; IC95\%: 1,11; 1,98), família não apoiou a gestação ( $R P=1,68$; IC95\%: 1,20-2,35) e repetência escolar ( $\mathrm{RP}=0,706$; IC95\%: 0,52; 0,95). As adolescentes que pertencem à classe social $\mathrm{D}+\mathrm{E}$ apresentaram 2,2 vezes mais prevalência de sofrimento psíquico intenso do que aquelas que pertencem à classe social $\mathrm{C}$ e $\mathrm{B}$. Foi ainda observada 2 vezes mais prevalência de sofrimento psíquico intenso nas adolescentes que não manifestaram um bom relacionamento com suas mães do que naquelas com um bom relacionamento. Ademais, verificou-se 1,4 vezes mais prevalência de sofrimento psíquico intenso nas adolescentes que não tiveram o apoio do parceiro na gestação do naquelas que tiveram o apoio do parceiro. As adolescentes que não tiveram o apoio da família na gestação apresentaram 1,6 vezes mais prevalência de sofrimento psíquico intenso do que as adolescentes que tiveram o apoio da família na 
Tabela 1. Prevalência, razão de prevalência (RP) bruta e ajustada entre sofrimento psíquico intenso (SP), aspectos sociodemográficos (nível 1), relações familiares (nível 2), aspectos reprodutivos, abuso e violência (nível 3). Porto Alegre (RS), 2009-2010.

\begin{tabular}{|c|c|c|c|c|c|c|c|c|c|}
\hline \multirow[b]{2}{*}{ Variáveis } & \multicolumn{2}{|c|}{ Frequência } & \multicolumn{7}{|c|}{ Sofrimento Psíquico Intenso } \\
\hline & $\mathbf{n}$ & $\%$ & $\%$ & $\begin{array}{c}\mathrm{RP} \\
\text { bruta }\end{array}$ & (IC 95\%) & p-valor & $\begin{array}{c}\text { RP } \\
\text { Ajustada* }^{*}\end{array}$ & (IC 95\%) & p-valor \\
\hline \multicolumn{10}{|l|}{ Aspectos sociodemográficos } \\
\hline \multicolumn{10}{|l|}{ Raça $(\mathrm{n}=415)$} \\
\hline Branca & 203 & 48,9 & 36,5 & 1,267 & $(0,96 ; 1,67)$ & 0,097 & 1,232 & $(0,93 ; 1,63)$ & 0,141 \\
\hline Não Branca & 212 & 51,1 & 28,8 & 1 & & & 1 & & \\
\hline \multicolumn{10}{|l|}{ Classe social (n = 399) } \\
\hline $\mathrm{D}+\mathrm{E}$ & 132 & 33,1 & 40,2 & 1,957 & $(1,02 ; 3,76)$ & 0,043 & 2,292 & $(1,13 ; 4,65)$ & 0,021 \\
\hline $\mathrm{C}$ & 228 & 57,1 & 32 & 1,561 & $(0,82 ; 2,98)$ & 0,177 & 1,776 & $(0,88 ; 3,57)$ & 0,107 \\
\hline $\mathrm{B}$ & 39 & 9,8 & 20,5 & 1 & & & 1 & & \\
\hline \multicolumn{10}{|l|}{ Repetência escolar $(\mathrm{n}=421)$} \\
\hline Sim & 331 & 78,6 & 31,4 & 0,808 & $(0,60 ; 1,09)$ & 0,169 & 0,706 & $(0,52 ; 0,95)$ & 0,023 \\
\hline Não & 90 & 21,4 & 38,9 & 1 & & & 1 & & \\
\hline \multicolumn{10}{|l|}{ Relações sociais e familiares } \\
\hline \multicolumn{10}{|c|}{ Criação pela mãe biológica $(\mathrm{n}=426)$} \\
\hline Não & 49 & 11,5 & 36,7 & 1,145 & $(0,77 ; 1,70)$ & 0,504 & & & \\
\hline Sim & 377 & 88,5 & 32,1 & 1 & & & & & \\
\hline \multicolumn{10}{|c|}{ Mora com o marido $(n=426)$} \\
\hline Não & 175 & 41,1 & 33,1 & 1,027 & $(0,78 ; 1,35)$ & 0,850 & & & \\
\hline Sim & 251 & 58,9 & 32,3 & 1 & & & & & \\
\hline \multicolumn{10}{|c|}{ Relacionamento com o pai $(\mathrm{n}=352)$} \\
\hline Ruim & 138 & 39,2 & 37 & 1,163 & $(0,87 ; 1,56)$ & 0,313 & & & \\
\hline Bom & 214 & 60,8 & 31,8 & 1 & & & & & \\
\hline \multicolumn{10}{|c|}{ Relacionamento com a mãe $(\mathrm{n}=418)$} \\
\hline Ruim & 83 & 19,9 & 57,8 & 2,202 & $(1,70 ; 2,85)$ & $<0,001$ & 2,023 & $(1,56 ; 2,62)$ & $<0,001$ \\
\hline Bom & 335 & 80,1 & 26,3 & 1 & & & 1 & & \\
\hline \multicolumn{10}{|c|}{ Possui uma pessoa confidente $(n=426)$} \\
\hline Não & 75 & 17,6 & 41,3 & 1,343 & $(0,98 ; 1,83)$ & 0,064 & 1,174 & $(0,86 ; 1,59)$ & 0,303 \\
\hline Sim & 351 & 82,4 & 30,8 & 1 & & & 1 & & \\
\hline \multicolumn{10}{|c|}{ Aspectos reprodutivos, abuso e violência } \\
\hline \multicolumn{10}{|c|}{ Idade da primeira relação sexual } \\
\hline 10 a 12 & 31 & 7,3 & 51,6 & 1,976 & $(1,28 ; 3,04)$ & 0,002 & 1,231 & $(0,70 ; 2,17)$ & 0,472 \\
\hline 13 a 14 & 234 & 55,5 & 35 & 1,342 & $(0,98 ; 1,84)$ & 0,068 & 1,162 & $(0,84 ; 1,60)$ & 0,361 \\
\hline 15 a 16 & 157 & 37,2 & 26,1 & 1 & & & 1 & & \\
\hline \multicolumn{10}{|c|}{ Queria engravidar $(\mathrm{n}=423)$} \\
\hline Sim & 73 & 17,3 & 35,6 & 1,103 & $(0,78 ; 1,56)$ & 0,576 & & & \\
\hline Não & 350 & 82,7 & 32,3 & 1 & & & & & \\
\hline \multicolumn{10}{|c|}{ Parceiro aceitou a gestação $(n=403)$} \\
\hline Não & 89 & 22,1 & 43,8 & 1,546 & $(1,15 ; 2,07)$ & 0,004 & 1,483 & $(1,11 ; 1,98)$ & 0,008 \\
\hline Sim & 314 & 77,9 & 28,3 & 1 & & & 1 & & \\
\hline \multicolumn{10}{|c|}{ Família apoiou a gestação $(\mathrm{n}=404)$} \\
\hline Não & 41 & 10,1 & 61 & 2,088 & $(1,56 ; 2,80)$ & $<0,001$ & 1,678 & $(1,20 ; 2,35)$ & 0,003 \\
\hline Sim & 363 & 89,9 & 29,2 & 1 & & & 1 & & \\
\hline \multicolumn{10}{|c|}{ Sofreu abuso sexual $(n=426)$} \\
\hline Sim & 28 & 6,6 & 39,3 & 1,222 & $(0,75 ; 1,98)$ & 0,416 & & & \\
\hline Não & 398 & 93,4 & 32,2 & 1 & & & & & \\
\hline \multicolumn{10}{|c|}{ Sofreu abuso físico $(\mathrm{n}=426)$} \\
\hline Sim & 59 & 13,8 & 45,8 & 1,500 & $(1,09 ; 2,06)$ & 0,012 & 0,937 & $(0,66 ; 1,33)$ & 0,718 \\
\hline Não & 367 & 86,2 & 30,5 & 1 & & & 1 & & \\
\hline
\end{tabular}

IC95\%: intervalo de 95\% de confiança

* ajustada pelas variáveis significantes $(\mathrm{p} \leq 0,20)$ do nível 1 (raça, classe social e repetência escolar), do nível 2 (relacionamento com sua mãe e possui uma pessoa confidente) e demais variáveis do nível 3 (idade da primeira relação sexual, parceiro aceitou a gestação, família apoiou a gestação, sofreu abuso físico), excluindo-se aquelas com $\mathrm{p}>0,10$ após ajuste. 
gestação. A repetência escolar foi uma variável protetora para o sofrimento psíquico intenso apresentando 33\% menos prevalência de sofrimento psíquico do que a não repetência.

Quanto a pouca ou nenhuma expectativa em relação ao futuro (Tabela 2 ), as variáveis repetência escolar, criação pela mãe biológica, mora com o marido, idade da primeira relação sexual, desejo de engravidar, apoio da família na gestação e sofreu abuso físico atingiram significância estatística menor ou igual a 20\% na análise bivariada e foram inseridas na análise multivariada. Permaneceram no modelo final, com significância estatística observada menor a $5 \%$, as variáveis: repetência escolar ( $\mathrm{RP}=2,56$; IC95\%:1,34-4,90), não ter sido criada pela mãe biológica $(R P=0,36$; IC95\%:0,14-0,93), idade da primeira relação sexual (RP = 1,75; IC95\%:1,12-2,75) e sofreu abuso físico ( $\mathrm{RP}=1,66$; IC95\%:1,09-2,52). A prevalência de pouca ou nenhuma expectativa em relação ao futuro foi 2,5 vezes maior para as adolescentes que repetiram o ano escolar por uma ou mais vezes do que para as que não repetiram. Não ter sido criada por sua mãe biológica aparece como uma variável protetora com $60 \%$ menos prevalência da adolescente apresentar pouca expectativa em relação ao futuro do que aquelas criadas pela mãe biológica. As adolescentes que tiveram a sua primeira relação sexual em idades mais precoces (entre os 13 a 14 anos) apresentaram 1,7 vezes mais prevalência de pouca expectativa em relação ao futuro do que aquelas que tiveram a primeira relação sexual com 15 ou 16 anos e as que sofreram abuso físico apresentaram 1,6 vezes mais prevalência de pouca ou nenhuma expectativa em relação ao futuro do que as adolescentes que não sofreram.

A Tabela 3 apresenta os resultados da relação que se estabeleceu entre as variáveis preditoras estudadas com a autovalorização negativa. $\mathrm{Na}$ análise bivariada, as variáveis morar com o marido, relacionamento com o pai, relacionamento com a mãe, possuir uma pessoa confidente, idade da primeira relação sexual, parceiro aceitou a gestação, família apoiou a gestação e sofreu abuso sexual atingiram nível de significância menor ou igual a $20 \%$ e foram inseridas na análise multivariada. Após o ajuste para fatores de confusão, as variáveis que foram incluídas na análise multivariada com significância estatística observada menor do que 5\%, foram: o relacionamento ruim com a mãe ( $R P=2,01$; IC95\%: 1,25-3,24), e não possuir uma pessoa confidente $(\mathrm{RP}=1,75$; IC95\%: 1,07-2,86). As adolescentes que manifestaram um relacionamento ruim com suas mães apresentaram duas vezes mais prevalência de apresentar autovalorização negativa do que aquelas com um bom relacionamento; e aquelas que não tinham uma pessoa confidente durante a gestação apresentaram 1,7 vezes mais prevalência de apresentar autovalorização negativa do que as que tinham.

\section{Discussão}

O presente estudo procurou verificar a prevalência de indicadores emocionais negativos e fatores associados em uma amostra de mães adolescentes de 14 a 16 anos, que tiveram filhos no ano de 2009 em Porto Alegre, RS. Dos desfechos estudados, o que apresentou maior prevalência foi o sofrimento psíquico intenso $(32,6 \%)$, seguido de autovalorização negativa $(15,4 \%)$ e de pouca ou nenhuma expectativa em relação ao futuro $(7,5 \%)$. Este resultado sinaliza um quadro desfavorável, em que uma a cada três adolescentes puérperas apresenta sofrimento psíquico.

Sabroza et al. ${ }^{9}$ pesquisaram 1.228 mães de 12 a 19 anos do Município do Rio de Janeiro, seguindo uma metodologia semelhante à do presente estudo, e encontraram prevalência de $26,8 \%$ para o sofrimento psíquico intenso. Freitas e Botega ${ }^{17}$, ao investigar transtornos psíquicos em 120 adolescentes grávidas de 14 a 18 anos em Piracicaba (SP), identificaram prevalências de $23,3 \%$ para ansiedade, 20,8\% para depressão e 16,7\% para ideação suicida. Caputo e Bordin ${ }^{18}$, ao compararem 207 adolescentes primigestas e 308 adolescentes não grávidas, apontaram que as grávidas apresentaram maior prevalência de sintomas de depressão e ansiedade (24,2\% vs 15,3\%) e retraimento/depressão ( $13 \%$ vs 4,5\%) do que as jovens não grávidas.

Os resultados do presente estudo, assim como os resultados das pesquisas mencionadas acima, indicam a necessidade de atentar para os aspectos afetivos/emocionais das adolescentes já durante a gestação, visto que estes podem acarretar prejuízos tanto para a adolescente como também para seu bebê. Por exemplo, a revisão de literatura realizada por Silva e Camargo ${ }^{19}$ mostra que, ao experienciar consequências psicológicas negativas, a adolescente grávida pode sofrer problemas gestacionais que podem afetar diretamente sua saúde, como casos de pré-eclampsia e diabetes gestacional, da mesma forma que a saúde do bebê como prematuridade e sofrimento fetal. Também Brum e Schermann ${ }^{13}$ afirmam que essas repercussões negativas podem afetar não apenas 
Tabela 2. Prevalência, razão de prevalência (RP) bruta e ajustada entre pouca ou nenhuma expectativa em relação ao futuro (ERF) e aspectos sociodemográficos (nível 1), relações familiares (nível 2), aspectos reprodutivos, abuso e violência (nível 3), Porto Alegre (RS), 2009-2010.

\begin{tabular}{|c|c|c|c|c|c|c|c|c|c|}
\hline \multirow[b]{2}{*}{ Variáveis } & \multicolumn{2}{|c|}{ Frequência } & \multicolumn{7}{|c|}{ Pouca ou nenhuma expectativa em relação ao futuro } \\
\hline & $\mathbf{n}$ & $\%$ & $\%$ & $\begin{array}{c}\text { RP } \\
\text { bruta }\end{array}$ & (IC 95\%) & p-valor & $\begin{array}{c}\text { RP } \\
\text { Ajustada* }^{*}\end{array}$ & (IC 95\%) & p-valor \\
\hline \multicolumn{10}{|l|}{ Aspectos sociodemográficos } \\
\hline \multicolumn{10}{|l|}{$\operatorname{Raça}(\mathrm{n}=416)$} \\
\hline Branca & 204 & 49 & 8,3 & 0,870 & $(0,60 ; 1,26)$ & 0,456 & & & \\
\hline Não Branca & 212 & 51 & 6,1 & 1 & & & & & \\
\hline \multicolumn{10}{|l|}{ Classe social } \\
\hline $\mathrm{D}+\mathrm{E}$ & 133 & 33,2 & 7,5 & 1,382 & $(0,66 ; 2,88)$ & 0,387 & & & \\
\hline $\mathrm{C}$ & 229 & 57,1 & 6,6 & 1,143 & $(0,56 ; 2,34)$ & 0,714 & & & \\
\hline B & 39 & 9,7 & 10,3 & 1 & & & & & \\
\hline \multicolumn{10}{|l|}{ Repetência escolar $(n=423)$} \\
\hline Sim & 331 & 78,3 & 8,8 & 2,563 & $(1,34 ; 4,90)$ & 0,004 & 2,332 & $(1,22 ; 4,45)$ & 0,010 \\
\hline Não & 92 & 21,7 & 3,3 & 1 & & & 1 & & \\
\hline \multicolumn{10}{|c|}{ Relações sociais e familiares } \\
\hline \multicolumn{10}{|c|}{ Criação pela mãe biológica $(\mathrm{n}=428)$} \\
\hline Não & 48 & 11,2 & 4,2 & 0,360 & $(0,14 ; 0,94)$ & 0,036 & 0,364 & $(0,14 ; 0,93)$ & 0,035 \\
\hline Sim & 380 & 88,8 & 7,9 & 1 & & & 1 & & \\
\hline \multicolumn{10}{|c|}{ Mora com o marido $(\mathrm{n}=428)$} \\
\hline Não & 175 & 40,9 & 6,9 & 0,735 & $(0,50 ; 1,08)$ & 0,118 & 0,714 & $(0,48 ; 1,04)$ & 0,083 \\
\hline Sim & 253 & 59,1 & 7,9 & 1 & & & 1 & & \\
\hline \multicolumn{10}{|c|}{ Relacionamento com o pai $(\mathrm{n}=354)$} \\
\hline Ruim & 137 & 38,7 & 7,3 & 0,937 & $(0,62 ; 1,41)$ & 0,755 & & & \\
\hline Bom & 217 & 61,3 & 7,4 & 1 & & & & & \\
\hline \multicolumn{10}{|c|}{ Relacionamento com a mãe $(n=420)$} \\
\hline Ruim & 82 & 19,5 & 11 & 1,073 & $(0,69 ; 1,67)$ & 0,756 & & & \\
\hline Bom & 338 & 80,5 & 6,5 & 1 & & & & & \\
\hline \multicolumn{10}{|c|}{ Possui uma pessoa confidente $(\mathrm{n}=428)$} \\
\hline Não & 76 & 17,8 & 10,5 & 1,287 & $(0,84 ; 1,98)$ & 0,250 & & & \\
\hline Sim & 352 & 82,2 & 6,8 & 1 & & & & & \\
\hline \multicolumn{10}{|c|}{ Aspectos reprodutivos, abuso e violência } \\
\hline \multicolumn{10}{|c|}{ Idade da primeira relação sexual $(n=424)$} \\
\hline 10 a 12 & 31 & 7,3 & 12,9 & 2,302 & $(1,21 ; 4,37)$ & 0,011 & 1,421 & $(0,67 ; 3,01)$ & 0,358 \\
\hline 13 a 14 & 236 & 55,7 & 7,2 & 1,814 & $(1,16 ; 2,83)$ & 0,009 & 1,752 & $(1,12 ; 2,75)$ & 0,015 \\
\hline 15 a 16 & 157 & 37 & 7 & 1 & & & 1 & & \\
\hline \multicolumn{10}{|l|}{ Queria engravidar $(\mathrm{n}=405)$} \\
\hline Sim & 74 & 17,4 & 8,1 & 1,423 & $(0,94 ; 2,16)$ & 0,098 & 1,085 & $(0,68 ; 1,73)$ & 0,734 \\
\hline Não & 351 & 82,6 & 7,4 & 1 & & & 1 & & \\
\hline \multicolumn{10}{|c|}{ Parceiro aceitou a gestação $(n=405)$} \\
\hline Não & 89 & 22 & 11,2 & 1,130 & $(0,73 ; 1,74)$ & 0,579 & & & \\
\hline Sim & 316 & 78 & 7 & 1 & & & & & \\
\hline \multicolumn{10}{|c|}{ Família apoiou a gestação $(\mathrm{n}=406)$} \\
\hline Não & 41 & 10,1 & 19,5 & 1,585 & $(0,97 ; 2,60)$ & 0,067 & 1,564 & $(0,99 ; 2,48)$ & 0,057 \\
\hline Sim & 365 & 89,9 & 6,6 & 1 & & & 1 & & \\
\hline \multicolumn{10}{|c|}{ Sofreu abuso sexual $(\mathrm{n}=428)$} \\
\hline Sim & 28 & 6,5 & 10,7 & 1,176 & $(0,60 ; 2,30)$ & 0,634 & & & \\
\hline Não & 400 & 93,5 & 7,2 & 1 & & & & & \\
\hline \multicolumn{10}{|c|}{ Sofreu abuso físico $(\mathrm{n}=428)$} \\
\hline Sim & 59 & 13,8 & 8,5 & 1,628 & $(1,07 ; 2,49)$ & 0,024 & 1,660 & $(1,09 ; 2,52)$ & 0,018 \\
\hline Não & 369 & 86,2 & 7,3 & 1 & & & 1 & & \\
\hline
\end{tabular}

IC95\%: intervalo de 95\% de confiança.

*ajustada pelas variáveis significantes $(\mathrm{p} \leq 0,20)$ do nível 1 (repetência escolar), do nível 2 (criação pela mãe biológica, mora com o marido), e demais variáveis do nível 3 (idade da primeira relação sexual, queria engravidar, família apoiou a gestação, sofreu abuso físico), excluindo-se aquelas com p >0,10 após ajuste. 
Tabela 3. Prevalência, razão de prevalência (RP) bruta e ajustada entre baixa autovalorização (AV), aspectos sociodemográficos (nível 1) relações sociais e familiares (nível 2), aspectos reprodutivos, abuso e violência (nível 3). Porto Alegre (RS), 2009-2010.

\begin{tabular}{|c|c|c|c|c|c|c|c|c|c|}
\hline \multirow[b]{2}{*}{ Variáveis } & \multicolumn{2}{|c|}{ Frequência } & \multicolumn{7}{|c|}{ Autovalorização negativa } \\
\hline & $\mathbf{n}$ & $\%$ & $\%$ & $\begin{array}{c}\text { RP } \\
\text { bruta }\end{array}$ & (IC 95\%) & p-valor & $\begin{array}{c}\text { RP } \\
\text { Ajustada }^{*}\end{array}$ & (IC 95\%) & p-valor \\
\hline \multicolumn{10}{|l|}{ Aspectos sociodemográficos } \\
\hline \multicolumn{10}{|l|}{ Raça $(\mathrm{n}=417)$} \\
\hline Branca & 205 & 49,2 & 17,1 & 1,340 & $(0,84 ; 2,13)$ & 0,216 & & & \\
\hline Não Branca & 212 & 50,8 & 12,7 & 1 & & & & & \\
\hline \multicolumn{10}{|l|}{ Classe social $(\mathrm{n}=402)$} \\
\hline $\mathrm{D}+\mathrm{E}$ & 133 & 33,1 & 18 & 1,173 & $(0,52 ; 2,66)$ & 0,703 & & & \\
\hline $\mathrm{C}$ & 230 & 57,2 & 12,6 & 0,820 & $(0,36 ; 1,84)$ & 0,631 & & & \\
\hline B & 39 & 9,7 & 15,4 & 1 & & & & & \\
\hline \multicolumn{10}{|l|}{ Repetência escolar $(\mathrm{n}=424)$} \\
\hline Sim & 332 & 78,3 & 16 & 1,130 & $(0,64 ; 1,98)$ & 0,670 & & & \\
\hline Não & 92 & 28,7 & 14,1 & 1 & & & & & \\
\hline \multicolumn{10}{|l|}{ Relações sociais e familiares } \\
\hline \multicolumn{10}{|c|}{ Criação pela mãe biológica $(\mathrm{n}=429)$} \\
\hline Não & 49 & 11,4 & 20,4 & 1,385 & $(0,76 ; 2,53)$ & 0,290 & & & \\
\hline Sim & 380 & 88,6 & 14,7 & 1 & & & & & \\
\hline \multicolumn{10}{|c|}{ Mora com o marido $(\mathrm{n}=429)$} \\
\hline Não & 176 & 41 & 18,8 & 1,437 & $(0,92 ; 2,24)$ & 0,108 & 1,340 & $(0,84 ; 2,13)$ & 0,216 \\
\hline Sim & 253 & 59 & 13 & 1 & & & 1 & & \\
\hline \multicolumn{10}{|c|}{ Relacionamento com o pai $(\mathrm{n}=355)$} \\
\hline Ruim & 138 & 38,9 & 21 & 1,629 & $(1,01 ; 2,61)$ & 0,043 & 1,516 & $(0,94 ; 2,43)$ & 0,085 \\
\hline Bom & 217 & 61,1 & 12,9 & 1 & & & 1 & & \\
\hline \multicolumn{10}{|c|}{ Relacionamento com a mãe $(\mathrm{n}=421)$} \\
\hline Ruim & 83 & 19,7 & 28,9 & 2,443 & $(1,56 ; 3,81)$ & $<0,001$ & 2,012 & $(1,25 ; 3,24)$ & 0,004 \\
\hline Bom & 338 & 80,3 & 11,8 & 1 & & & & & \\
\hline \multicolumn{10}{|c|}{ Possui uma pessoa confidente $(n=429)$} \\
\hline Não & 76 & 17,7 & 27,6 & 2,168 & $(1,37 ; 3,42)$ & 0,001 & 1,749 & $(1,07 ; 2,86)$ & 0,026 \\
\hline Sim & 353 & 82,3 & 12,7 & 1 & & & & & \\
\hline \multicolumn{10}{|c|}{ Aspectos reprodutivos, abuso e violência } \\
\hline \multicolumn{10}{|c|}{ Idade da primeira relação sexual $(\mathrm{n}=425)$} \\
\hline 10 a 12 & 31 & 7,3 & 22,6 & 1,970 & $(0,90 ; 4,31)$ & 0,090 & 1,196 & $(0,45 ; 3,20)$ & 0,723 \\
\hline 13 a 14 & 237 & 55,8 & 16 & 1,398 & $(0,83 ; 2,36)$ & 0,209 & 1,017 & $(0,58 ; 1,78)$ & 0,954 \\
\hline 15 a 16 & 157 & 36.9 & 11,5 & 1 & & & 1 & & \\
\hline \multicolumn{10}{|l|}{ Queria engravidar $(n=426)$} \\
\hline Sim & 74 & 17,4 & 16,2 & 1,057 & $(0,60 ; 1,87)$ & 0,849 & & & \\
\hline Não & 352 & 82,6 & 15,3 & 1 & & & & & \\
\hline \multicolumn{10}{|c|}{ Parceiro aceitou a gestação $(n=406)$} \\
\hline Não & 90 & 22,2 & 23,3 & 1,940 & $(1,20 ; 3,13)$ & 0,007 & 1,561 & $(0,92 ; 2,64)$ & 0,096 \\
\hline $\operatorname{Sim}$ & 316 & 77,8 & 12,0 & 1 & & & 1 & & \\
\hline \multicolumn{10}{|c|}{ Família apoiou a gestação $(\mathrm{n}=407)$} \\
\hline Não & 41 & 10,1 & 29,3 & 2,100 & $(1,22 ; 3,60)$ & 0,007 & 1,312 & $(0,67 ; 2,57)$ & 0,429 \\
\hline Sim & 366 & 89,9 & 13,9 & 1 & & & & & \\
\hline \multicolumn{10}{|c|}{ Sofreu abuso sexual $(n=429)$} \\
\hline Sim & 28 & 6,5 & 28,6 & 1,975 & $(1,05 ; 3,72)$ & 0,035 & 1,277 & $(0,50 ; 3,28)$ & 0,613 \\
\hline Não & 401 & 93,5 & 14,5 & 1 & & & & & \\
\hline \multicolumn{10}{|c|}{ Sofreu abuso físico $(\mathrm{n}=429)$} \\
\hline $\operatorname{Sim}$ & 59 & 13,8 & 20,3 & 1,394 & $(0,79 ; 2,44)$ & 0,247 & & & \\
\hline Não & 370 & 86,2 & 14,6 & 1 & & & & & \\
\hline
\end{tabular}

IC95\%: intervalo de 95\% de confiança.

* ajustada pelas variáveis significantes $(\mathrm{p} \leq 0,20)$ do nível 2 (mora com o marido, relacionamento com o pai, relacionamento com a mãe, possui ma pessoa confidente), e demais variáveis do nível 3 (idade da primeira relação sexual, parceiro aceitou a gestação, família apoiou a gestação, sofreu abuso sexual), excluindo-se aquelas com $\mathrm{p}>0,10$ após ajuste. 
a função materna da adolescente como também a qualidade do vínculo mãe-bebê, fundamental para o desenvolvimento da criança.

Pode-se reconhecer que certo nível de sofrimento psíquico e de baixa autovalorização são características presentes na fase de desenvolvimento da adolescência em razão de todas as transformações corporais, cognitivas e sociais que ocorrem nessa etapa vital. Porém, como salientam Freitas e Botega ${ }^{17}$, esses aspectos emocionais parecem acentuar-se na ocorrência de uma gestação e, assim, desencadear um quadro de depressão.

No presente estudo, houve maior representatividade de mães adolescentes das classes das classes C (57\%) e D+E (33\%), havendo apenas 10\% da amostra pertencente à classe $\mathrm{B}$ e nenhuma da classe A. A associação da condição socioeconômica com o sofrimento psíquico intenso ficou evidenciado nas adolescentes das classes de menor nível socioeconômico (D+E) quando comparadas às mães adolescentes da classe social B e C. Duarte et $\mathrm{al}^{8}{ }^{8}$ encontraram maior prevalência de gestação em adolescentes com piores condições socioeconômicas, enquanto que menor prevalência de gestação mostrou-se associada com melhores condições socioeconômicas entre as 1.314 adolescentes investigadas em Santo André (SP).

Os dados obtidos no presente estudo mostraram que a maioria das adolescentes $(78,6 \%)$ repetiram o ano letivo em algum momento de suas vidas, sendo que em $70 \%$ destes casos, houve repetição de dois ou mais anos. Araújo et al. ${ }^{20}$ também constataram uma alta taxa de repetência $(69 \%)$ entre adolescentes grávidas de Juiz de Fora (MG). A questão da escolaridade vem sendo apontada por outros estudos empíricos tanto como fator de risco para a gravidez nessa faixa etária, como consequência da gravidez. Em relação ao fator de risco, Sipsma et al. ${ }^{21}$ sublinham que quando as adolescentes não frequentam a escola, o risco de engravidarem potencializa-se e, como consequência da gestação, muitas adolescentes acabam abandonando ou interrompendo os estudos. Por exemplo, Aquino et al. ${ }^{3}$ verificaram que, das 4.364 participantes, $25 \%$ interromperam temporariamente os estudos e $17,3 \%$ abandonaram os estudos definitivamente no primeiro ano após o nascimento do bebê. Silva et al. ${ }^{22}$ salientam que a baixa escolaridade também está associada à gravidez recorrente na adolescência.

No presente estudo, a repetência escolar aparece como um fator de risco para a expectativa em relação ao futuro, mas como um fator de proteção para o sofrimento psíquico. Pode-se pensar que, para as adolescentes estudadas, o fato de estudar adequadamente e não repetir o ano seria considerado mais estressante do que repeti-lo, protegendo-as, portanto, do sofrimento ocasionado pela necessidade de dedicação para o estudo. A alta prevalência de repetência escolar parece confirmar esta suposição. Por outro lado, a repetência escolar pode também ter sido ocasionada pela gravidez e/ou cuidados com o bebê recém-nascido, que, neste momento tornam-se prioridade. $\mathrm{O}$ contraponto aparece na consciência das adolescentes de que a repetência escolar constitui-se um fator importante para o seu futuro.

As adolescentes que manifestaram bom relacionamento com a mãe apresentaram sofrimento psíquico menos intenso e autovalorização mais alta do que aquelas com relacionamento ruim com as mães. O relacionamento entre mãe e filha foi investigado em vários estudos. A figura materna é uma fonte de identificação para a filha, especialmente quando esta também se torna mãe e o bom relacionamento com a própria mãe pode auxiliar a superar dificuldades ${ }^{23}$. Lima et al. ${ }^{24}$ estudaram as percepções e as práticas de adolescentes grávidas e de familiares em relação à gestação e mostraram que, entre as 19 adolescentes entrevistadas, $71,5 \%$ têm a mãe como responsável pelo seu cuidado e $57,2 \%$ contam com o apoio afetivo e financeiro materno. Diante da gravidez das filhas, embora o sentimento das mães tenha variado entre alegria-satisfação $(35,7 \%)$ e desgosto $(28,5 \%)$, a maioria (93\%) aceitou o fato. Da mesma forma, Schwartz et al. ${ }^{10}$, em estudo qualitativo com 12 adolescentes gestantes, apontaram a mãe como uma importante fonte de apoio afetivo e material para as participantes. Junto com a mãe, estes mesmos estudos salientam a importância do apoio do parceiro da adolescente (pai do bebê). Por exemplo, Lima et al. ${ }^{24}$ demonstraram que $37 \%$ das adolescentes recorreram, primeiramente, ao seu parceiro quando descobriram a gravidez. Schwartz et al. ${ }^{10}$ afirmam que, para quatro das 12 adolescentes entrevistadas, o parceiro foi a principal fonte de apoio. No presente estudo, 78\% das adolescentes afirmaram que tiveram a aceitação do parceiro em relação à gravidez, e aquelas que não a tiveram apresentaram 1,5 vezes mais chance de sofrimento psíquico intenso.

Pedrosa et al..$^{25}$ verificaram que as adolescentes que se sentiam menos apoiadas pelo parceiro e pela mãe apresentaram maiores dificuldades durante a gravidez. O apoio social é fundamental para uma experiência mais positiva da maternidade, especialmente para as mães adolescen- 
tes $^{13,15}$. As dificuldades que surgem na gravidez adolescente podem ser minimizadas pelo apoio social recebido ${ }^{18}$. Sabe-se, por exemplo, que a adolescente ao engravidar vivencia uma série de expectativas em relação à gestação e ao nascimento do bebê, e, muitas vezes, essas expectativas geram medo e ansiedade, que são amenizados e até superados quando há $\mathrm{o}$ amparo familiar ${ }^{10,12}$. $\mathrm{O}$ fato de ter o apoio de uma pessoa confidente torna-se igualmente importante para a mãe adolescente, gerando uma melhor autovalorização, conforme o demonstrado neste estudo.

Nessa direção, os resultados da presente pesquisa indicam a importância da aceitação da gravidez pela família de modo a provocar um menor sofrimento psíquico. Das adolescentes estudadas, $90 \%$ afirmaram ter recebido o apoio de sua família. Em pesquisa com 22 adolescentes, Witter ${ }^{26}$ analisou a percepção de adolescentes grávidas em relação aos seus familiares e parceiros, evidenciando que, para $41 \%$ das adolescentes, os pais reagiram mal à notícia da gravidez, porém depois aceitaram, $36 \%$ dos pais reagiram de modo normal e $9 \%$ reagiram bem.

A gravidez adolescente pode gerar sentimentos ambíguos nos familiares desde o choque com a notícia, a decepção pela não prevenção e a frustração pela interrupção de planos e projetos de vida, até a aceitação e a alegria pela chegada de um bebêế $\hat{e}^{27}$. Além disso, a gravidez provoca um nível significativo de mudanças familiares, que podem ser percebidas de diferentes maneiras por seus membros $^{28}$. Lewandowski et al. ${ }^{29}$ asseveram que, em geral, as famílias das gestantes e das mães adolescentes acabam apoiando-as, porém existem situações de rejeição, que podem ser decorrentes de fatores sociais específicos. No presente estudo, a reação negativa da família em relação à gestação da adolescente foi associada com o sofrimento psíquico intenso. Sabroza et al. ${ }^{9}$, analisando essa mesma variável, mostraram associação deste fator com os três desfechos estudados.

A expectativa em relação ao futuro tem um papel importante para as mães adolescentes, visto que as motiva a buscarem melhores condições para suas vidas e a de seus filhos, com uma projeção mais positiva frente ao futuro e a possibilidade de criação de um projeto de vida. Os resultados do presente estudo apontam que, além da repetência escolar mencionada anteriormente, a idade precoce da primeira relação sexual, ter sofrido abuso físico em algum momento de suas vidas e não ter sido criada pela mãe biológica foram associados a pouca expectativa em relação ao futuro para as mães adolescentes.
Em relação à idade da primeira relação sexual, encontrou-se que 55,7\% das adolescentes relataram ter a primeira relação entre os 13 e 14 anos. Esse número é semelhante ao encontrado por Aquino et al. ${ }^{3}$, que entrevistou 4.634 jovens em três capitais brasileiras. Dentre estas, $52,8 \%$ eram do sexo feminino e $69,7 \%$ tinham atividade sexual antes dos 14 anos de idade. O início precoce de vida sexual pode representar um fator de risco para o desenvolvimento emocional saudável ${ }^{30-32}$

Outro fator que parece dificultar uma prospecção positiva diante do futuro é o sofrimento de abuso físico. Em estudo ${ }^{32}$ com 776 crianças investigadas por um período de 17 anos em Teresina (PI), constatou-se que adolescentes com histórico de maus tratos na infância são três vezes mais propensos a tornarem-se deprimidos ou tentarem suicídio quando comparados aos participantes sem antecedentes.

Vieira et al..$^{33}$ investigaram o perfil de crianças e adolescentes vítimas de violência física e seus resultados revelaram que, em sua maioria, as crianças e os adolescentes tiveram como agressores os próprios pais, destacando as mães $(45,28 \%)$, seguido dos pais $(32,07 \%)$. Pode-se pensar que essas adolescentes que sofreram abuso físico, especialmente no contexto familiar, possuem menor possibilidade de receberem apoio da família durante a gestação, fator já relatado como necessário para uma repercussão mais positiva da gravidez na vida da adolescente.

Não ter sido criada pela mãe biológica mostrou-se, no presente estudo, como um fator protetor de expectativa em relação ao futuro, sendo apresentado por $11 \%$ das adolescentes. No estudo de Caputo e Bordin ${ }^{5}$ com adolescentes de 13 a 17 anos grávidas e não grávidas, os autores afirmam que o fato da adolescente ter sido criada por outra pessoa, que não pela mãe biológica, pode apontar para problemas familiares, como a mãe ter deixado o bebe com a avó por ser muito nova para cuidar da criança, separação do casal seguido de abandono da criança pela mãe ou mesmo morte desta. Neste sentido, a criação por outra pessoa que não a mãe, pode constituir-se de um maior benefício emocional para a criança.

Por fim, os resultados do presente estudo apontam para a influência de diferentes aspectos para cada um dos desfechos estudados, sendo o relacionamento da adolescente com sua mãe e a repetência escolar os mais evidenciados. A alta prevalência de sofrimento psíquico, encontrada nas mães adolescentes deste estudo, merece atenção especial das políticas públicas de saúde para a inclusão de profissionais habilitados ao manejo 
dos aspectos emocionais no atendimento da maternidade precoce. Melo e Coelho ${ }^{34}$ indicam em sua pesquisa inserir no cotidiano dos profissionais, processos educativos grupais que ofereçam suporte para as adolescentes desenvolverem habilidades para a vida, tais como negociação, comunicação, resolução de conflitos e tomada de decisões.

É importante ressaltar algumas limitações desta pesquisa. Por se tratar de um estudo trans- versal, ele não pode ser utilizado para estabelecer relações causais definitivas. Além disso, os resultados do estudo estão baseados em pontos de corte do quartil (25\%) da distribuição da frequência dos desfechos analisados. Outros pontos de corte podem apresentar resultados diferenciados dos aqui apresentados. Ainda, algumas variáveis, por sua estratificação e baixa prevalência, podem ter resultado em baixo poder estatístico, devendo os resultados serem interpretados com cautela.

\section{Colaboradores}

MS Rossetto realizou a fundamentação teórica, correção do banco de dados, análise e interpretação dos dados, redação do artigo e aprovação final da versão a ser submetida para publicação. LB Schermann e JU Béria participaram da concepção e delineamento do projeto mais amplo, treinamento da equipe de coleta de dados, elaboração do banco de dados, revisão do artigo e aprovação final da versão a ser submetida para publicação. LB Schermann participou ainda como orientadora no método da pesquisa, análise e interpretação dos dados.

\section{Agradecimentos}

Ao Conselho Nacional de Desenvolvimento Científico e Tecnológico ( $\mathrm{CNPq}$ ), pelo financiamento da pesquisa. 


\section{Referências}

1. World Health Organization (WHO). Guidelines on Preventing Early Pregnancy and Poor Reproductive Outcomes Among Adolescents in Developing Countries. [acessado 2011 nov 16]. Disponível em: http:// www.who.int/ making_pregnancy_safer/topics/adolescent_pregnancy/en/index.html

2. Brasil. Ministério da Saúde (MS). Gravidez na adolescência - Saúde do adolescente e do jovem. [acessado 2011 jul 14]. Disponível em: http://portal.saude.gov. $\mathrm{br} /$ portal/arquivos/multimedia /adolescente/principal. htm

3. Aquino EML, Heiborn ML, Knauth D, Bozon M, Almeida MC, Araújo J, Menezes G. Adolescência e reprodução no Brasil: a heterogeneidade dos perfis sociais. Cad Saude Publica 2003; (Supl. 2):S377-S388.

4. Calligaris C. A adolescência. 2a ed. São Paulo: Publifolha; 2009.

5. Caputo VG, Bordin IA. Problemas de saúde mental entre jovens grávidas e não-grávidas. Rev Saude Publica 2007; 41(4):573-581.

6. Silva AA, Camargo NC. Repercussões negativas de gravidez na adolescência: Revisão de literatura. Rev Cient Eletr Psicol 2008; Ano VI(11).

7. Esteves JR, Menandro PRM. Trajetórias de vida: Repercussões da maternidade adolescente na biografia de mulheres que viveram tal experiência. Universidade Federal do Rio Grande do Norte. Estud Psicol 2010; 10(3):363-370.

8. Duarte CM, Nascimento VB, Akerman M. Gravidez na adolescência e exclusão social: análise de disparidades intra-urbanas. Rev Panam Salud Publica 2006; 19(4):236-243.

9. Sabroza AR, Leal MC, Junior PRS, Goma SGN. Algumas repercussões emocionais negativas de gravidez precoce com adolescentes do Município do Rio de Janeiro. Cad Saude Publica 2004; 20(Supl. 1):130-137.

10. Schwartz T, Vieira R, Geib LTC. Apoio social a gestantes: desvelando percepções. Cien Saude Colet 2011; 16(5):2575-2585.

11. Piccinini CA, Rapoport A, Levandowski DC, Voigt PR. Apoio social percebido entre mães adolescentes e adultas. (Porto Alegre). Psico 2002; 33(1):9-35.

12. Godinho RA, Schelp JRB, Parada CMGL, Bertoncello NMF. Adolescentes e grávidas: onde buscam apoio? Rev Latinoam Enferm 2000; 8(2):25-32.

13. Brum EHM, Shermann LB. Intervenção para promover a qualidade do vínculo mãe-bebê em situação de nascimento pré-termo. Rev Bras Crescimento Desenvolv Hum 2007; 17(2):12-23.

14. Brum EHM, Shermann LB. O impacto da depressão materna nas interações iniciais. (Porto Alegre). Psico 2006; 37(2):151-158.

15. Coutinho LMS, Scazufca M, Menezes PR. Métodos para estimar razão de prevalências em estudos de corte transversal. Rev Saude Publica 2008; 42(6):992-998.

16. Brasil. Ministério da Saúde (MS). Conselho Nacional de Saúde. Resolução nº 196 de 10 de outubro de 1996. Diretrizes e Normas Regulamentadoras de Pesquisas Envolvendo Seres Humanos. Diário Oficial da União 1996; 16 out.

17. Freitas GVS, Botega NJ. Gravidez na adolescência: prevalência de depressão, ansiedade e ideação suicida. Rev Assoc Med Bras 2002; 48(3):245-249.
18. Caputo VG, Bordin IA. Problemas de saúde mental entre jovens grávidas e não-grávidas. Rev Saude Publica 2007; 41(4):573-581.

19. Silva AA, Camargo NC. Repercussões negativas de gravidez na adolescência: Revisão de literatura. Rev Cient Eletr Psicol 2008; Ano VI(11).

20. Araújo DAC, Laporte BEP, Silva JA, Zimmermmam JB, Vieira CM, Oliveira L, Andrade ATL. Perfil socioeconômico de gestantes adolescentes. Bol Centro Biol Reprod 2002; 21:45-58.

21. Sipsma HL, Ickovics JR, Lewis JB, Ethier KA, Kersshaw TS. Adolescent Pregnancy Desire and Pregnancy Incidence. Women's Health Issues 2010; 21(2):110-116.

22. Silva KS, Rozenberg R, Bonan C, Chuva VCC, Costa SF, Gomes MASM. Gravidez recorrente na adolescência e vulnerabilidade social no Rio de Janeiro (RJ, Brasil): uma análise de dados do Sistema de Nascidos Vivos. Cien Saude Colet 2011; 16(5):2485-2493.

23. Maldonado MT. Psicologia da Gravidez, Parto e Puerpério. Rio de Janeiro: Ed. Saraiva; 1997.

24. Lima CTB, Feliciano KVO, Carvalho MFS, Souza APP, Menabó JBC, Ramos SL, Cassundé LF, Kovacs MH. Percepções e práticas de adolescentes grávidas e de familiares em relação à gestação. Rev Bras Saúde Matern Infant 2004; 4(1):71-83.

25. Pedrosa AA, Pires R, Carvalho P, Canavarro MC, Dattilio F. Ecological Contexts in Adolescent Pregnancy: The role of individual, sociodemographic, Familial and relational variables in understanding risk of ocurrence and adjustment paterns. Contemp Fam Ther 2011; 33:107-127.

26. Witter GP. Percepção de adolescentes grávidas em relação a seus familiares e parceiros. Psicol Cienc Prof 2008; 28(3):548-557.

27. Silva L, Tonete VLP. A gravidez na adolescência sob a perspectiva dos familiares: compartilhando projetos de vida e cuidado. Rev Latinoam Enferm 2006; 14(2):199206.

28. East PL, Chien NC. Family dynamics across pregnant Latina Adolescents transition to parenthood. J Fam Psychol 2010; 24(6):709-720.

29. Levandowski DC, Piccinini CA, Lopes RCS. Maternidade adolescente. (Campinas) Estud Psicol 2008; 25(2):251-263.

30. Dimbuene ZT, Defo BK. Family environment and premarital intercourse in Bandjoun (West Cameroon). Arch Sex Behav 2012; 41(2):351-361.

31. Graaf H, Schoot R, Woertman L, Hawk S, Meeus W. Family Cohesion and Romantic and sexual initiation: A three wave longitudinal study. J Youth Adolesc 2012; 41(5):583.

32. Monteiro CFS, Costa NSS, Nascimento PSV, Aguiar YA. A violência intra-familiar contra adolescentes grávidas. Rev Bras Enferm 2007; 60(4):373-376.

33. Vieira D, Martins CS, Ferriani MGC, Nascimento LC. Caracterização da violência física contra crianças e adolescentes. Rev Enferm UERJ 2004; 12(3):306-311.

34. Melo MCP, Coelho EAC. Integralidade e cuidado a grávidas adolescentes na Atenção Básica. Cien Saude Colet 2011; 16(5):2549-2558.

Artigo apresentado em 18/07/2013

Aprovado em 17/06/2014

Versão final apresentada em 25/06/2014 